\title{
3 Research Square

\section{Health Literacy Knowledge Level of Chinese Registered Nurses and Associated Practices: A Cross-sectional Survey}

\section{Ying Qian}

Zhejiang University School of Medicine Sir Run Run Shaw Hospital https://orcid.org/0000-0002-47599968

Jingjie Wu

Zhejiang University School of Medicine

\section{Lili Yang (D 3200006@zju.edu.cn)}

Zhejiang University School of Medicine Sir Run Run Shaw Hospital

\section{Xiaofang Jiang}

Zhejiang University School of Medicine Sir Run Run Shaw Hospital

\section{Huaizeng Chen}

Zhejiang University School of Medicine Women's Hospital

\section{Research article}

Keywords: Health literacy, Nursing knowledge, Registered nurse, Health education

Posted Date: March 17th, 2021

DOI: https://doi.org/10.21203/rs.3.rs-301712/v1

License: (c) (1) This work is licensed under a Creative Commons Attribution 4.0 International License. Read Full License 


\section{Abstract}

\section{Background}

Health literacy is recognized as a social determinant of health based on its impact on health outcomes. Nurses play a vital role in the promotion of health literacy, health literacy practices enable nurses to offer understandable health information to all people and contribute to health equity. The aims of this study were to investigate health literacy knowledge level and associated practices among Chinese registered nurses and identify related factors.

\section{Methods}

A cross-sectional study design was used. The Chinese version of the Health Literacy Knowledge and Experience Survey was adapted among registered nurses $(N=768)$ from six hospitals in Zhejiang Province of China. Descriptive statistics, one-way analysis of variance and multiple linear regression were used to analyze the data of demographic characteristics, knowledge and associated practices.

\section{Results}

The health literacy knowledge level of 768 nurses was $12.78 \pm 3.54$, of the five content areas, basic facts about health literacy had the lowest correct answer rate 43.2\%. Educational background $(P=0.000)$, position ( $P=0.010)$, professional title $(P=0.030)$, department $(P=0.009)$, and whether they had heard about health literacy $(P=0.022)$ were significantly correlated with health literacy knowledge level. The multiple linear regression analysis found that educational background and whether they had heard about health literacy were positively associated with health literacy knowledge level. In the aspect of health literacy education practice, using written materials to provide healthcare information $(P=0.000)$, using computer software to provide healthcare information $(P=0.003)$ and using teach-back to examine the effect of health education $(P=0.000)$ were positively correlated with health literacy knowledge level.

\section{Conclusion}

This study indicated that registered nurses in Zhejiang Province of China had low health literacy knowledge level and applied health literacy-associated practices inadequately. It is urgent to improve the knowledge level of nurses' health literacy. Educational background, position, professional title, department, and whether they had heard about health literacy were significantly correlated with health literacy knowledge level. In the continuous training, it is very important to design corresponding health literacy courses according to different departments and different levels of nurses.

\section{Background}

Health literacy $(\mathrm{HL})$ is "a dynamic, collaborative, and mutually beneficial proficiency incorporating prior health knowledge and experience, individual characteristics, health status, cultural and linguistic preferences, and cognitive abilities influencing the ability of organizations, caregivers, and health care 
recipients to access, understand and use health information and services to make informed actionable decisions and enhance health outcomes" [1]. Many studies have indicated that limited HL is associated with poor health-related knowledge and comprehension, poor health status and high utilization of health care services, including hospital and emergency care, which increase the economic burden on families and society [2, 3]. In the United States, more than one-third of the population has limited HL [4] and an estimated 90 million Americans struggle to understand recommendations made by health care professionals (HCPs) [3]. In China, only $28 \%$ of residents had adequate health literacy knowledge in Beijing and just $19.3 \%$ of residents in Wuhan $[5,6]$.

HCPs play an important role in providing useful health knowledge to the public $[7,8]$. Nurses are the largest group of HCPs. Therefore, they are fully capable of improving the health literacy of patients by providing understandable health knowledge. However, some studies indicate that nurses have little knowledge and a negative attitude toward $\mathrm{HL}[9,10]$, which can be an obstacle for the implementation of effective $\mathrm{HL}$-associated practice. Nurses use a variety of methods to improve communication with patients and consider the lack of the concept of $\mathrm{HL}$ and the neglect of $\mathrm{HL}$ in HCPs as obstacles to its evaluation at higher levels than do physicians [11].

In China, health literacy was included as an evaluation indicator for healthy living in "Healthy China 2030"strategic planning [12]. Enhancing public health literacy is an important strategy to improve the overall health status of a population, but to the best of our knowledge, no study on registered nurses (RNs)' HL knowledge level and associated practices has been reported in China. The aims of this study were to investigate $\mathrm{HL}$ knowledge level and associated practices and identify their related factors among Chinese RNs.

\section{Methods}

\section{Design}

This study used a cross-sectional design to survey RNs of Zhejiang Province in China about their HL knowledge level and associated practices and their related factors.

\section{Sample and setting}

The RNs working in six hospitals in Zhejiang Province of China (two county hospitals, two municipal hospitals, and two university hospitals) were invited using convenient sampling to participate in this study. After obtaining permission to conduct the survey from each hospital, questionnaires were distributed by the respective nursing managers. Nursing students and none bedside nurses were excluded from the study. There were 816 responses, of which 768 (94.1\%) were complete.

\section{Data collection}


The nurse managers of the included hospitals, and a coordinator at the Nurse Education Department at one of the hospitals, handed out a questionnaire through WeChat to the RNs. The survey was anonymous, no coding system to identify respondents was used. No reminder was given. Data were collected between June and August 2019.

\section{Questionnaire}

We adapted the Health Literacy Knowledge and Experience Survey (HL-KES) [13], to the Chinese population through standard procedures [14] and then used the Chinese version of HL-KES to collect data. The final questionnaire consisted of three sections: section 1: demographic characteristics, including gender, age, work experience, educational background, hospital level, position, professional title, department, awareness of $\mathrm{HL}$, and previous $\mathrm{HL}$ training. Section 2: participants' knowledge of $\mathrm{HL}$ (comprised 27 multiple choice questions in five content areas), including basic facts about HL (5 multiple choice questions); consequences associated with low HL (4 multiple choice questions); HL screening (6 multiple choice questions); guidelines for written healthcare materials (9 multiple choice questions) and evaluation of $\mathrm{HL}$ interventions (3 multiple choice questions). The Cronbach's alpha and $\mathrm{KMO}$ values were 0.527 and 0.667 respectively. Section 3: the HL experiences of participants during their nursing careers. And participants were asked to respond to $7 \mathrm{HL}$ experience questions rated by selecting one of four choices: Never, Sometimes, Frequently, and Always. The Cronbach's alpha and KMO values were 0.804 and 0.824 .

\section{Date analysis}

Means, standard deviations (SDs), and correct answer rate were calculated for $\mathrm{HL}$ knowledge level. Descriptive statistics, including frequencies and proportions, were calculated to describe demographic characteristics. One-way analysis of variance (ANOVA) was used to analyze differences of knowledge levels among demographic characteristics and $\mathrm{HL}$ experiences. Multiple linear regression was used to assess the associations between $\mathrm{HL}$ knowledge and demographic characteristics. Frequencies and proportions were calculated for the $\mathrm{HL}$ experience data. Statistics analyses employed the Statistical Package for Social Science (SPSS), version 17.0. Statistical significance level was set at $p<0.05$.

\section{Ethical approval}

All ethical concerns of the Helsinki Declaration were followed. Ethical approval for the study was granted by the Institutional Review Board of Zhejiang University School of Medicine Sir Run Run Shaw Hospital. RNs were informed that (a) their participation was voluntary, (b) they could refuse to answer any item, and (c) there would be no adverse consequences for refusal. Signed informed consent to participate was obtained from all subjects. No participant-identifiable data was recorded to maintain confidentiality. 


\section{Results}

\section{Knowledge levels of $\mathrm{HL}$ in five content areas}

The mean value of the HL knowledge scores of 768 nurses was $12.78 \pm 3.54$. Of the five content areas, basic facts about $\mathrm{HL}$ had the lowest correct answer rate was $43.2 \%$, while evaluation of $\mathrm{HL}$ interventions had the highest correct answer rate was $51.0 \%$ (shown in Table 1).

\section{Demographic Characteristics and their Relationships with HL Knowledge}

As shown in Table 2, survey data indicated that $50.9 \%$ of RNs had never heard of $\mathrm{HL}$, and $86.1 \%$ of those never attended $\mathrm{HL}$ training. Gender $(P=0.013)$, educational background $(P=0.000)$, position $(P=0.010)$, professional title $(P=0.030)$, department $(P=0.009)$, and whether they had heard about $\mathrm{HL}(P=0.022)$ were significantly correlated with $\mathrm{HL}$ knowledge level. However, there was no significant correlation between age, working experience, hospital level and whether attended HL training or not with HL knowledge level.

The multiple linear regression analysis found that educational background and whether they had heard about HL were positively associated with HL knowledge level (Shown in Table 3).

\section{HL Experience and the relationships with HL knowledge}

The responses regarding HL experience are provided in Table 4. Using written materials to provide healthcare information $(P=0.000)$, using computer software to provide healthcare information $(P=0.003)$ and using teach-back to examine the effect of health education $(P=0.000)$ were positively correlated with HL knowledge level.

\section{Discussion}

$\mathrm{HL}$ is recognized as a social determinant of health based on its impact on health outcomes. Nurses play a vital role in the promotion of $\mathrm{HL}, \mathrm{HL}$ practices enable nurses to offer understandable health information to all people and contribute to health equity. Nurses, as educators, are obligated to evaluate the patients' health literacy and find strategies to improve patients' health literacy. Furthermore, nurses' health literacy knowledge is essential to the quality of health education. Nurses' lack of health literacy knowledge can lead to reduced effectiveness of health education, blocked public access to health information, a further expansion of the gap in $\mathrm{HL}$ among the population, and finally detriment to health-related outcomes. Recent studies show that in the United States, the knowledge of health literacy for nursing students and practicing nurses is obviously insufficient, and their experience in health literacy activities is very limited [15]. 
Our study supported the previous findings in the United States as we found that RNs in China had low HL knowledge level, especially regarding basic HL facts, which had the lowest correct answer rate. 50.9\% nurses had never heard of $\mathrm{HL}$ and $86.1 \%$ never attended $\mathrm{HL}$ training. Macabasco-O'Connell A's study also revealed that nursing professionals' knowledge of $\mathrm{HL}$ and their understanding on the role $\mathrm{HL}$ plays on patient health outcomes is limited [16]. Nevertheless, some studies reported better circumstance, for example, Knight et al. found that RNs in Georgia had some HL knowledge. Three of the six basic facts on health literacy items were answered correctly by the majority of participants but three were also answered incorrectly by the majority of participants [17]. Low HL knowledge level may be caused by various factors. Our study was able to find that HL education background may have relationship with health literacy knowledge level. Ozen found that the $\mathrm{HL}$ of almost one-third of nursing students was limited, likely due to a lack of HL courses related in their 4-year curriculum [18]. In China, some universities set up mandatory or optional health education and health promotion courses in undergraduate program. In fact, $\mathrm{HL}$ education is also very scarce in continuing education program in hospitals. That explained why there was no significant difference in HL knowledge among nurses in different levels of hospitals in our study. Above findings suggested that $\mathrm{HL}$ education was essential for nursing group.

Our findings indicate that nurse manager, nurse educator and nurses with higher professional titleNational career ladder for nurses: level 1-5) have a higher level of HL knowledge level, possibly because they have more willingness to receive HL knowledge and learning resources. Nurses from different departments also showed gaps in HL knowledge. HL training for nurses in Health examination centers and Outpatient department needs to be strengthened. However, there was no statistical significance in whether or not they had attended HL training. It indicates that the effectiveness of $\mathrm{HL}$ education is not enough, which may be the problem of educational methods, educational content, or teaching ability of teachers. Kaper et al. reported that $\mathrm{HL}$ communication training for HCPs enhanced their knowledge and skills to improve patients' autonomy in decision-making and strengthened their intention to employ HL-related communication [19]. Nurse educators and leaders could use the HL Tapestry conceptual model in education and practice to describe and define $\mathrm{HL}$ across the continuum of care [20]. Thus, well-designed and comprehensive HL education programs integrated into the nurse curriculum in universities and continuous training in hospitals are urgently needed. At the same time, it can choose senior title's nurses and nurse managers as teachers, and further improve their knowledge of $\mathrm{HL}$. Develop individualized training programs to meet the training needs of nurses in different departments.

Regarding HL-associated practices, most nurses lacked the ability to evaluate a patient's level of HL with a HL screening tool. This is closely related to the lack of popularization of HL screening tools, such as the Test of Functional Health Literacy in Adults (TOFHLA) and Rapid Estimate of Adult Literacy in Medicine (REALM), in clinical applications. Currently, there are no rapid HL screening tools in clinical practice in China. Due to inadequate awareness of the popularization of $\mathrm{HL}$, resulting in limited $\mathrm{HL}$, less than half of nurses evaluate a patient's reading level and the cultural appropriateness of materials when providing health-related information. Most nurses assess patients' HL empirically. $27 \%$ of nurses never or rarely ask whether a patient has difficulty reading medical information or completing medical forms. A total of $60 \%$ 
of respondents stated they use their "gut feeling" to assess the patient's HL often or always [16]. Therefore, future studies should focus not only on the development of $\mathrm{HL}$ screening tools but also on the applicability of such tools in clinical settings. When selecting health education pathways, nurses can choose written materials, videotapes or computer software. A total of $47.5 \%$ of nurses always or frequently use the teach-back method to assess the effect of health education. Though nurses conduct these practices in everyday patient interactions, there is still room for improvement. As Loan et al. noted, the use of the HL Universal Precautions Toolkit (AHRQ) may be beneficial for all health care providers across a variety of settings, as it promotes clear verbal and written communication to support optimal outcomes in health care [20]. Therefore, nurses should adopt effective communication techniques, such as using simple language, using teach-back methods, writing or printing out instruction materials or drawing pictures, when conducting health education [21]. Our study found that the higher the level of nurses' HL knowledge, in practice, nurses do better in using written materials. But nurses who never using computer software to provide healthcare information and never using teach-back to examine the effect of health education have higher $\mathrm{HL}$ scores. Nurses need to use more modern and effective educational tools and methods to educate patients with $\mathrm{HL}$ knowledge, not only with written materials

There are certain limitations of this study that need to be discussed. First, selection bias cannot be excluded, as only 768 nurses in hospitals in Zhejiang Province of China were recruited. Therefore, the samples are not representative of the whole nation. Second, during the adaptation of the instrument, no pilot tests were conducted, which may reduce the reliability and validity of the questionnaire, but the Cronbach's alpha and KMO values still indicate that the adapted version has good reliability and validity.

\section{Conclusion}

This study indicated that RNs in Zhejiang Province of China had low HL knowledge level and applied HLassociated practices inadequately. It is urgent to improve the knowledge level of nurses' health literacy. Educational background, position, professional title, department, and whether they had heard about HL were significantly correlated with HL knowledge level. In the continuous training, it is very important to design corresponding health literacy courses according to different departments and different levels of nurses.

\section{Abbreviations}

HL: Health literacy

HCPs: Health Care Professionals

RNs: Registered Nurses

HL-KES: Health Literacy Knowledge and Experience Survey

SDs: Sandard Deviations 
ANOVA: Analysis of Variance

SPSS: Statistical Package for Social Science

TOFHLA: Test of Functional Health Literacy in Adults

REALM: Rapid Estimate of Adult Literacy in Medicine

\section{Declarations}

\section{Ethics approval and consent to participate}

Ethical approval for the study was granted by the Institutional Review Board of Zhejiang University School of Medicine Sir Run Run Shaw Hospital (Approval number: 20210108-30). Written consent was obtained from all participants.

\section{Consent for publication}

Not applicable.

\section{Availability of data and material}

The dataset analysed during the current study are available from the corresponding author on reasonable request.

\section{Competing interests}

The authors declare that they have no competing interests.

\section{Funding}

This research did not receive any specific grant from funding agencies in the public, commercial, or notfor-profit sectors.

\section{Authors' contributions}

YQ and LLY: Study design. XFJ: Data collection. YQ: Data analysis. YQ and JJW: Data interpretation. YQ and JJW: Contribution of the first draft. HZC and LLY: Final version approval. All Authors read and approved the manuscript. 


\section{Acknowledgements}

The authors would like to acknowledge the participation of nurses.

\section{References}

1. Parnell TA, Stichler JF, Barton AJ, Loan LA, Boyle DK, Allen PE. A concept analysis of health literacy. Nurs Forum. 2019;54(3):315-27.

2. Pelikan JM, Ganahl K, Roethlin F. Health literacy as a determinant, mediator and/or moderator of health: empirical models using the European Health Literacy Survey dataset. Glob Health Promot. 2018:1757975918788300.

3. Berkman ND, Sheridan SL, Donahue KE, Halpern DJ, Crotty K. Low health literacy and health outcomes: an updated systematic review. Ann Intern Med. 2011;155(2):97-107.

4. Hersh L, Salzman B, Snyderman D. Health Literacy in Primary Care Practice. American family physician. 2015;92(2):118-124.

5. Shi J, Qi L, Li Y, Liu X. Investigation of Health Literacy Status in Beijing, China. Health Lit Res Pract. 2020;4(3):e174-e84.

6. Mei X, Zhong Q, Chen G, Huang Y, Li J. Exploring health literacy in Wuhan, China: a cross-sectional analysis. BMC Public Health. 2020;20(1):1417.

7. Chang LC, Chen YC, Wu FL, Liao LL. Exploring health literacy competencies towards patient education programme for Chinese-speaking healthcare professionals: a Delphi study. BMJ Open. 2017;7(1):e011772.

8. Coleman C, Hudson S, Pederson B. Prioritized Health Literacy and Clear Communication Practices For Health Care Professionals. Health Lit Res Pract. 2017;1(3):e91-e9.

9. Rajah R, Hassali MA, Lim CJ. Health Literacy-Related Knowledge, Attitude, and Perceived Barriers: A Cross-sectional Study among Physicians, Pharmacists, and Nurses in Public Hospitals of Penang, Malaysia. Front Public Health. 2017;5:281.

10. Nesari M, Olson JK, Nasrabadi AN, Norris C. Registered Nurses' Knowledge of and Experience with Health Literacy. Health Lit Res Pract. 2019;3(4):e268-e79.

11. Guner MD, Ekmekci PE. A Survey Study Evaluating and Comparing the Health Literacy Knowledge and Communication Skills Used by Nurses and Physicians. The Journal of Health Care Organization Provision and Financing. 2019, 56:0046958019865831.

12. The State Council of China. Healthy China 2030 strategic planning.2016.Retrieved from http://www.nhc.gov.cn/guihuaxxs/s3586s/201610/21d120c917284007ad9c7aa8e9634bb4.shtml

13. Cormier CM, Kotrlik JW. Health Literacy Knowledge and Experiences of Senior Baccalaureate Nursing Students. J Nurs Educ. 2009;48(5):237-48.

14. Sousa VD, Rojjanasrirat W. Translation, adaptation and validation of instruments or scales for use in cross-cultural health care research: a clear and user-friendly guideline. J Eval Clin Pract. 
2011;17(2):268-74.

15. Cafiero M. Nurse practitioners' knowledge, experience, and intention to use health literacy strategies in clinical practice. J Health Commun. 2013;18 Suppl 1:70-81.

16. Macabasco-O'Connell A, Fry-Bowers EK. Knowledge and perceptions of health literacy among nursing professionals. J Health Commun. 2011;16 Suppl 3:295-307.

17. Knight GD. An Evaluation of the Health Literacy Knowledge and Experience of Registered Nurses in Georgia. Auburn University. 2011.

18. Ozen N, Bal Ozkaptan B, Coskun S, Terzioglu F. Health literacy of nursing students and its effective factors. Nurs Forum. 2019;54(3):396-402.

19. Kaper, M. S. , Sixsmith, J. , Koot, J. A. R. , Meijering, L. B. , Van Twillert, S. , \& Giammarchi, C. , et al. (2017). Developing and pilot testing a comprehensive health literacy communication training for health professionals in three european countries. Patient Education \& Counseling.101(1), 152-158.

20. Loan LA, Parnell TA, Stichler JF, Boyle DK, Allen P, VanFosson CA, et al. Call for action: Nurses must play a critical role to enhance health literacy. Nurs Outlook. 2018;66(1):97-100.

21. Koo LW, Horowitz AM, Radice SD, Wang MQ, Kleinman DV. Nurse Practitioners' Use of Communication Techniques: Results of a Maryland Oral Health Literacy Survey. Plos One. 2016;11(1).

\section{Tables}

Due to technical limitations, tables are only available as a download in the Supplemental Files section.

\section{Supplementary Files}

This is a list of supplementary files associated with this preprint. Click to download.

- Tables.pdf 